\title{
Análise do poder discriminatório da SE-AFLP para Salmonella Enteritidis frente a outras técnicas fenotípicas e genotípicas
}

\author{
Comparison of the discriminatory power of SE-AFLP for Salmonella \\ Enteritidis with other phenotypic and genotypic techniques \\ André Felipe Streck ${ }^{1}$, Clarissa Silveira Luiz Vaz ${ }^{1}$, Fernanda Simone Marks ${ }^{1}$, \\ Sílvia Dias de Oliveira ${ }^{2}$, Marisa Ribeiro de Itapema Cardoso $^{3} \&$ Cláudio Wageck Canal $^{1}$
}

\begin{abstract}
RESUMO
Salmonella $(S$.$) Enteritidis tem sido uma das bactérias mais implicadas em casos de infecções alimentares, sendo a$ investigação de sua epidemiologia realizada por diferentes métodos fenotípicos e genotípicos. Dentre as técnicas de tipificação de bactérias, a técnica de single-enzyme amplified fragment length polymorphism (SE-AFLP) é um dos métodos mais recentemente descritos, apresentando boa confiabilidade e fácil aplicação. No presente trabalho, a SE-AFLP foi comparada com as técnicas fenotípicas de fagotipificação (PT) e determinação da sensibilidade a antimicrobianos (DSA) e genotípicas de rep-PCR (seqüências repetitivas REP, ERIC e BOX) e detecção de genes de virulência (genes $s p v \mathrm{R}$ e $s p v \mathrm{C}$ ). Foram analisadas 20 amostras de $S$. Enteritidis, sendo onze isoladas de suínos da Região Sul do Brasil e nove amostras oriundas de outros países. A caracterização destas amostras pelas técnicas de PT, DSA, presença de genes de virulência e rep-PCR foi descrita em um trabalho anterior. O poder discriminatório obtido pelas técnicas foi calculado pelo índice de diversidade de Simpson $(D)$. A SE-AFLP encontrou um número maior de perfis e obteve uma maior capacidade discriminatória do que as outras técnicas genotípicas, embora seu $D$ tenha sido menor do que o das técnicas fenotípicas. Desta forma, ficou demonstrada a importância da utilização conjunta de técnicas fenotípicas e genotípicas na caracterização de amostras de $S$. Enteritidis.
\end{abstract}

Descritores: Salmonella Enteritidis, SE-AFLP, genotipagem, suíno, epidemiologia.

\section{ABSTRACT}

Salmonella $(S$.) Enteritidis is one of the most reported bacteria in outbreaks of food borne disease and its epidemiology has been investigated by different phenotyping and genotyping based methods. Fingerprinting by SE-AFLP is one of the most recent methods, presenting easy application and reproducibility. In the present study, the SE-AFLP method was compared with two phenotyping techniques; phage typing (PT) and antimicrobial susceptibility (DSA) and two genotyping techniques; repPCR (repetitive sequences REP, ERIC and BOX) and detection of virulence genes (genes $s p v \mathrm{R}$ and $s p v \mathrm{C}$ ). For these, 20 samples were analyzed, being eleven of swine origin from the Southern Region of Brazil and nine samples from other countries. The techniques of phage typing, DSA, presence of virulence genes and rep-PCR were performed in a previous study. For the accomplishment of the SE-AFLP, the technique was performed using the restriction enzyme Hind III. The discriminatory power obtained by the techniques was calculated by the Simpson's index of diversity $(D)$. Between the genotypic based techniques, the SEAFLP evidenced a higher number of profiles and obtained a best discriminatory capacity. However, the $D$ was lower than both phenotypic based techniques. The results evidenced the importance of the combined use of phenotypic and genotypic techniques for Salmonella Enteritidis typing.

Key words: Salmonella Enteritidis, SE-AFLP, genotyping, swine, epidemiology. 


\section{INTRODUÇÃO}

A salmonelose é uma das zoonoses de maior importância no mundo devido a perdas econômicas causadas na produção animal e por sua implicação em saúde pública [27,28]. Dentre os mais de 2000 sorovares desta bactéria, o Enteritidis ( $S$. Enteritidis), após a década de 90, passou a ser o mais isolado de alimentos de origem animal e surtos de infecção alimentar em seres humanos [2,17,23,25,27].

Medidas efetivas de controle da $S$. Enteritidis podem ser estabelecidas identificando sua fonte de contaminação e rastreando epidemiologicamente o isolado implicado dentro da cadeia produtiva animal. Tradicionalmente, esta investigação epidemiológica tem se baseado em características fenotípicas [10], porém, estas técnicas possuem algumas limitações. Nos últimos anos, o desenvolvimento da biologia molecular levou ao estabelecimento de novos métodos de tipagem baseados no genótipo de microrganismos [10]. Entre estas, um dos mais recentes métodos de tipificação por PCR, o single-enzyme amplified fragment length polymorphism (SE-AFLP), vem apresentando um ótimo potencial de discriminação [7,22].

Baseado nisto, o objetivo deste estudo foi avaliar a capacidade discriminatória da técnica de SE-AFLP para a caracterização de isolados de $S$. Enteritidis, comparando esta técnica com técnicas fenotípicas e outras técnicas genotípicas que haviam sido executadas em um trabalho anterior com estas mesmas amostras [20].

\section{MATERIAIS E MÉTODOS}

\section{Amostras utilizadas}

Foram analisadas 20 amostras de $S$. Enteritidis, sendo onze oriundas de carne e derivados de suínos e nove isoladas de surtos de infecção alimentar em humanos. As amostras suinícolas foram coletadas na Região Sul do Brasil (Rio Grande do Sul e Santa Catarina) durante o período de 1995 a 2001. As amostras de humanos foram isoladas em outros países (Albânia, Egito, Itália, Tanzânia e Zimbábue), não havendo informação sobre o ano de isolamento. Todas as amostras utilizadas eram epidemiologicamente não-relacionadas e haviam sido previamente isoladas, sorotipificadas e fagotipificadas [20]. Como controle das técnicas moleculares, foi utilizada a amostra 13076 da American type culture collection (ATCC).

\section{Determinação da sensibilidade a antimicrobianos, detecção de genes de virulência e rep-PCR}

As técnicas de determinação da sensibilidade a antimicrobianos, detecção de genes de virulência e
rep-PCR foram realizadas em trabalho anterior [20]. A DSA foi realizada conforme preconizado pelo NCCLS [18]. A sensibilidade das amostras foi testada frente aos seguintes antimicrobianos: ácido nalidíxico (30 mg), ampicilina (10 mg), cefalotina (30 mg), ciprofloxacina (5 mg), cloranfenicol (30 mg), gentamicina (10 mg), estreptomicina $(10 \mathrm{mg})$, nitrofurantoína $(300 \mathrm{mg})$ norfloxacina (10 mg), tetraciclina (30 mg) e sulfonamida (300 mg). A detecção de genes de virulência foi realizada através de PCR, com iniciadores para os genes $s p v \mathrm{R}$ e $s p v \mathrm{C}$. A técnica de rep-PCR foi realizada utilizando iniciadores para REP (REP1R-I e REP2-I), ERIC (ERIC1R e ERIC2) e BOX (BOX1R).

\section{SE-AFLP}

O método genotípico utilizado foi o SE-AFLP $[7,22]$ modificado. Para avaliação desta técnica, o DNA total foi obtido de culturas incubadas por $24 \mathrm{~h}$ a $37^{\circ} \mathrm{C}$ conforme protocolo anteriormente descrito [21]. Alíquotas contendo $4 \mu \mathrm{g}$ de DNA foram clivadas com $2 \mathrm{U}$ de Hind III $^{1}$, utilizando tampão específico da enzima, num volume final de $80 \mu \mathrm{L}$. A reação de ligação foi realizada a $16^{\circ} \mathrm{C}$, contendo $5 \mu \mathrm{L}$ de DNA clivado, $0,2 \mu \mathrm{g}$ de cada oligonucleotídeo adaptador ${ }^{2}, 1 \mathrm{U}$ de T4 DNA ligase $^{1}$ e tampão específico da enzima, em um volume final de $20 \mu \mathrm{L}$. O DNA ligado foi precipitado e ressuspenso em $10 \mathrm{mM}$ Tris- $\mathrm{HCl}$ pH 8,0 com 1 mM EDTA. Os fragmentos foram amplificados por PCR, onde foram utilizados quatro iniciadores que diferiram no último oligonucleotídeo da extremidade 3', sendo A, T, C ou G (referidos como iniciadores A, T, C e G). A PCR foi realizada utilizando-se $2,5 \mathrm{mM} \mathrm{MgCl}_{2}, 20 \mathrm{pmol}$ de um único iniciador (A, T, C ou G) e 1 U de Taq DNA Polimerase. A eletroforese foi realizada em gel de agarose ${ }^{3} 1,5 \%$. Os géis foram corados com brometo de etídio $(0,5 \mu \mathrm{g} / \mathrm{mL})$ e visualizados em transluminador de luz ultra-violeta. A SE-AFLP foi realizada com todas as amostras duas vezes.

\section{Análise dos fragmentos}

O poder discriminatório $(D)$ foi determinado pelo índice de diversidade de Simpson, que calcula a probabilidade da técnica de tipagem assinalar como distintas duas amostras não relacionadas e randomicamente selecionadas de uma população [8].

\section{RESULTADOS}

O número de produtos de amplificação por perfil de SE-AFLP variou de quatro a doze, situando-se entre 200 pares de bases (pb) e 2400 pb (Figura 1). 
Apenas as bandas situadas entre $200 \mathrm{pb}$ e $1800 \mathrm{pb}$ foram analisadas. Os iniciadores testados apresentaram um perfil predominante para a maioria das amostras (A1, C1, G1 e T1). Individualmente, foram obtidos dois perfis diferentes com o iniciador $\mathrm{T}$, quatro perfis com os iniciadores $\mathrm{A}$ e $\mathrm{G}$ e cinco perfis com o iniciador C. Os perfis não predominantes incluíram apenas uma ou duas amostras. Os resultados desta técnica e sua relação com as demais técnicas estão expostos na Tabela 1.

Ao analisar o poder discriminatório pelo índice de diversidade de Simpson, a SE-AFLP gerou o menor $D$ com o iniciador T $(0,19)$ e o maior com o C $(0,37)$, com uma média do índice de diversidade de Simpson obtido, com todos os iniciadores, igual a 0,28. Comparando a SE-AFLP com as demais técnicas genotípicas testadas, rep-PCR e a presença de genes de viru-

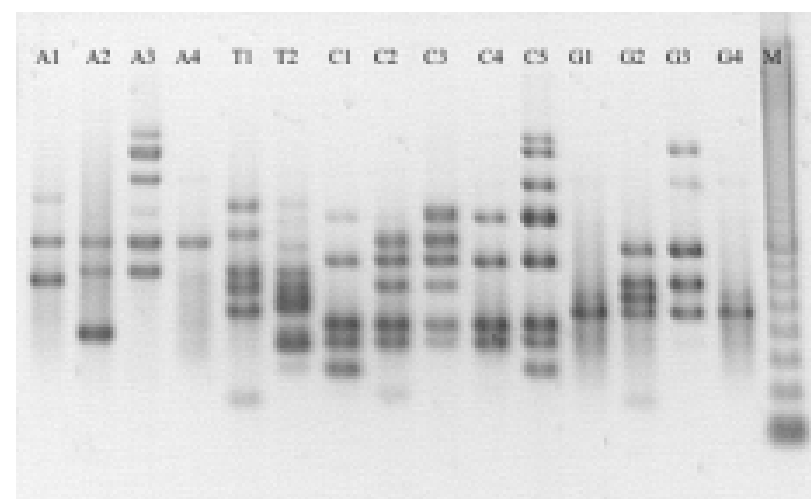

Figura 1. Visualização da eletroforese realizada em gel de agarose $1,5 \%$ dos produtos da SE-AFLP, com os perfis distintos obtidos com os iniciadores A (A1, A2, A3 e A4), T (T1 e T2), C (C1, C2, C3, C4 e C5) e G (G1, G2, G3 e G4). Obs.: mesmo tamanho, seguindo no parágrafo. M: marcador molecular de $100 \mathrm{pb}$.

Tabela 1. País e origem das amostras analisadas, juntamente com o fagotipo (PT), determinação da sensibilidade a antimicrobianos (DSA)*, perfil de REP, ERIC e BOX-PCR, presença de genes de virulência ( $s p v$ R e $s p v C$ ) e perfil obtido pelos diferentes iniciadores utilizados na técnica de SE-AFLP (A, C, G ou T).

\begin{tabular}{|c|c|c|c|c|c|c|c|c|c|c|c|c|}
\hline Núm. & País & PT & DSA $^{*}$ & REP & ERIC & BOX & spvR & spvC & AFLPa & AFLPc & AFLPg & AFLPt \\
\hline 01 & Brasil & $\mathrm{Nd}$. & Str, Nalx & $\mathrm{R} 1$ & $\mathrm{E} 1$ & $\mathrm{~B} 1$ & + & + & $\mathrm{A} 1$ & C1 & G1 & T1 \\
\hline 02 & Brasil & $6 a$ & Nit & R1 & $\mathrm{E} 1$ & B1 & + & + & $\mathrm{A} 1$ & C1 & G1 & T1 \\
\hline 03 & Brasil & 6 & Smx, Str, Nit, Ampe & $\mathrm{R} 1$ & $\mathrm{E} 1$ & B1 & + & + & $\mathrm{A} 1$ & $\mathrm{C} 1$ & G1 & T1 \\
\hline 04 & Brasil & $6 a$ & Str, Tet, Gen, Nit & $\mathrm{R} 1$ & E1 & B1 & + & + & $\mathrm{A} 1$ & $\mathrm{C} 1$ & G1 & $\mathrm{T} 1$ \\
\hline 05 & Brasil & $6 a$ & Smx, Str, Tet, Gen & $\mathrm{R} 1$ & $\mathrm{E} 1$ & B1 & + & + & A1 & $\mathrm{C} 1$ & G1 & T1 \\
\hline 06 & Brasil & $\mathrm{Nd}$ & Smx, Nit & R1 & E1 & B1 & + & + & A2 & $\mathrm{C} 2$ & G2 & T2 \\
\hline 07 & Brasil & $6 a$ & Str, Tet, Gen, Nit & R1 & $\mathrm{E} 1$ & B1 & + & + & $\mathrm{A} 1$ & $\mathrm{C} 1$ & G1 & T1 \\
\hline 08 & Brasil & $\mathrm{Nd}$ & Chl, Smx, Tet, Nit, Nalx & $\mathrm{R} 1$ & $\mathrm{E} 1$ & B2 & - & - & A3 & C3 & G3 & T2 \\
\hline 09 & Brasil & $7 a$ & Nit & R1 & $\mathrm{E} 1$ & B1 & + & + & A1 & C1 & G1 & T1 \\
\hline 10 & Brasil & $6 a$ & Str, Nit & $\mathrm{R} 1$ & $\mathrm{E} 1$ & B1 & + & + & $\mathrm{A} 1$ & C1 & G1 & T1 \\
\hline 11 & Brasil & $\mathrm{Nd}$ & Gen, Nit & R1 & $\mathrm{E} 1$ & B1 & + & + & $\mathrm{A} 1$ & $\mathrm{C} 1$ & G1 & $\mathrm{T} 1$ \\
\hline 12 & Tanzânia & 4 & Smx & R1 & E1 & B1 & + & + & $\mathrm{A} 1$ & $\mathrm{C} 1$ & G1 & T1 \\
\hline 13 & Zimbábue & 4 & - & $\mathrm{R} 1$ & $\mathrm{E} 1$ & B1 & + & + & $\mathrm{A} 1$ & $\mathrm{C} 1$ & G1 & T1 \\
\hline 14 & Itália & 11 & Nit & R1 & E1 & B1 & + & + & $\mathrm{A} 1$ & $\mathrm{C} 1$ & G1 & $\mathrm{T} 1$ \\
\hline 15 & Egito & 4 & Smx, Str, Nit & R1 & E1 & B1 & + & + & $\mathrm{A} 1$ & C1 & G1 & $\mathrm{T} 1$ \\
\hline 16 & Albânia & 6 & Nit & $\mathrm{R} 1$ & $\mathrm{E} 1$ & B1 & + & + & $\mathrm{A} 1$ & C1 & G1 & $\mathrm{T} 1$ \\
\hline 17 & Itália & 11 & Nit & R1 & $\mathrm{E} 1$ & B1 & + & + & $\mathrm{A} 1$ & $\mathrm{C} 1$ & G1 & $\mathrm{T} 1$ \\
\hline 18 & Tanzânia & 9 & Chl, Smx, Str, Ampe & $\mathrm{R} 2$ & E1 & B3 & + & + & A4 & $\mathrm{C} 4$ & G4 & $\mathrm{T} 1$ \\
\hline 19 & Albânia & 6 & - & $\mathrm{R} 1$ & $\mathrm{E} 1$ & B1 & + & + & $\mathrm{A} 1$ & $\mathrm{C} 1$ & G1 & $\mathrm{T} 1$ \\
\hline 20 & Zimbábue & 4 & Smx & R1 & $\mathrm{E} 1$ & B1 & + & + & $\mathrm{A} 1$ & $\mathrm{C} 5$ & G1 & $\mathrm{T} 1$ \\
\hline 21 & ATCC" & $\mathrm{Nd}$ & - & R1 & E3 & B1 & + & - & A1 & C1 & G1 & T1 \\
\hline $\mathrm{D}^{*}$ & & 0,83 & 0,93 & 0,10 & 0,00 & 0,19 & 0,10 & 0,10 & 0,28 & 0,37 & 0,28 & 0,19 \\
\hline
\end{tabular}

*Ácido nalidíxico (Nalx), ampicilina (Ampe), cefalotina (Cef), ciprofloxacina (Cipx), cloranfenicol (Chl), estreptomicina (Str), gentamicina (Gen), nitrofurantoína (Nit), norfloxacina (Nor), tetraciclina (Tet) e sulfonamida (Smx). ${ }^{* *}$ A amostra ATCC 13076 não foi considerada no cálculo de $D$. 
lência, observa-se que essas apresentaram um menor poder de discriminação para o grupo de isolados incluídos no estudo. Por sua vez, as técnicas fenotípicas, fagotipificação e DSA, apresentaram $D$ bastante superior a média obtida pela SE-AFLP.

\section{DISCUSSÃO}

Nos últimos anos, a $S$. Enteritidis tem sido a principal causadora de doenças transmitidas por alimentos no Estado do Rio Grande do Sul, gerando gastos públicos com hospitalização e perdas de dias de trabalho [2,17]. Por outro lado, o Brasil, na qualidade de país exportador, sofre pressões comerciais para que seus produtos de origem animal estejam livres deste patógeno, gerando a necessidade da implementação de tecnologias para seu controle. Diversas técnicas fenotípicas e genotípicas vêm sendo empregadas para a caracterização epidemiológica deste patógeno $[1,5,6,11,19]$. Porém, nestes estudos, existem poucas abordagens que ofereçam uma análise comparativa entre um número mais amplo de técnicas.

No presente trabalho, examinamos uma coleção de $S$. Enteritidis de origem suína e humana, coletadas no período entre 1995 e 2001 através da técnica de SE-AFLP. As técnicas de fagotipificação, determinação da sensibilidade a antimicrobianos, detecção de genes de virulência e rep-PCR, realizadas em estudo anterior com as mesmas amostras [20], foram utilizadas para comparação e fornecer suporte aos resultados obtidos com a SE-AFLP. Para realizar esta comparação, foi determinado o índice de diversidade de Simpson [8], que é frequentemente utilizado com esta finalidade [4,24].

A técnica de SE-AFLP apresentou um perfil que predominou na maioria das amostras com os quatro iniciadores (A1, C1, G1 e T1) testados. Além deste perfil predominante, também gerou alguns perfis distintos em algumas amostras com cada iniciador, resultando em quatro padrões com o iniciador $\mathrm{A}$ ou $\mathrm{G}$, cinco com o $\mathrm{C}$ e apenas dois com o $\mathrm{T}$. $\mathrm{O}$ valor de $D$ obtido foi de $0,28,0,37,0,28$ e 0,19 para os iniciadores A, C, G e T, respectivamente. Os métodos genotípicos de detecção de genes de virulência e rep-PCR também apresentaram, com todos seus iniciadores, a presença de um perfil predominante ( $s p v \mathrm{R}$ : presença; sp $v \mathrm{C}$ : presença; REP: R1; ERIC: E1 e BOX: B1). O valor de $D$ apresentado pelos iniciadores $s p v \mathrm{R}$ e $s p v \mathrm{C}$ foi de 0,10 para ambos, enquanto que os iniciadores de rep-PCR, REP, ERIC e BOX, apresentaram $D$ de
0,10, 0,00 e 0,19, respectivamente. Por sua vez, os métodos fenotípicos aplicados nestas amostras geraram um alto grau de diversidade. A DSA apresentou $D=$ 0,93 e a PT $D=0,83$. Ao todo, foram encontrados treze distintos perfis na técnica de DSA e seis perfis na técnica de PT, não sendo possível determinar a presença de um perfil predominante.

Os melhores resultados encontrados com a SE-AFLP frente às demais técnicas genotípicas referem-se, possivelmente, às diferenças de alvo das distintas técnicas. A rep-PCR baseia-se na amplificação de regiões entre seqüências repetitivas que se encontram esparsas no cromossomo bacteriano, sem função definida ou conhecida. Da mesma forma, a técnica de detecção de genes de virulência explora regiões gênicas específicas que, por sua importância para o patógeno, estão sujeitas a uma pequena variabilidade. A SE-AFLP, por sua vez, realiza uma análise indiscriminada em todo o cromossomo bacteriano, utilizando como alvo regiões que flanqueiam o sítio de clivagem da enzima de restrição (Hind III). Outra vantagem desta técnica sobre as demais é a utilização de quatro iniciadores (A, C, G e T), possibilitando encontrar um maior número de perfis, oriundos da diferença de um nucleotídeo na região de anelamento do iniciador. Já a diversidade de perfis e o valor de $D$ encontrados com as técnicas fenotípicas foram superiores inclusive à técnica de SE-AFLP. Entretanto, a expressão de um caráter fenotípico é suscetível de modificar-se quando as condições ambientais variarem [3], sendo, portanto, menos estável que o perfil genotípico.

A técnica de AFLP é um dos mais recentes métodos de tipagem molecular descritos [10] e vem sendo utilizada para o estudo epidemiológico de diversas bactérias [15,16,29,30]. Dentre as diferentes formas de realização da técnica de AFLP [9,14,29,32], a SE-AFLP é uma modificação da técnica que tem apresentado resultados confiáveis [7,22]. Como vantagens sobre a AFLP, é uma técnica mais simples de executar, e o fato de sua eletroforese poder ser realizada em gel de agarose proporciona sua aplicação em laboratórios de pequeno porte. Outra vantagem é que, por utilizar apenas uma enzima de restrição, a SE-AFLP gera padrões de bandas menos complexos que a AFLP, podendo ser interpretada sem o auxílio de programas específicos. Pelo seu custo mais acessível, facilidade de execução e interpretação de resultados, a SE-AFLP já foi considerada uma boa alternativa inclusive ao uso do PFGE, técnica padrão-ouro em tipagem atualmente [12,13]. 
Estudos recentes utilizando a SE-AFLP para caracterização de $S$. Enteritidis também verificaram a presença de boa capacidade discriminatória. Em estudo envolvendo amostras de $S$. Enteritidis de origem avícola, a SE-AFLP também gerou um perfil predominante, porém, conseguiu diferenciar todos os sorovares testados [31]. Ao analisar 172 isolados de $S$. Enteritidis de distintas origens, a técnica de SE-AFLP foi capaz de obter 12 distintos perfis [13], demonstrando possuir bom poder discriminatório.

No presente trabalho, a técnica de SE-AFLP obteve resultados superiores aos encontrados com as técnicas genotípicas que haviam sido empregadas anteriormente. Entretanto, os presentes resultados também indicam que as técnicas genotípicas testadas apresentam uma capacidade discriminatória limitada para isolados de $S$. Enteritidis epidemiologicamente não relacionados, confirmando o caráter clonal atribuído ao sorovar Enteritidis [23]. Por isto, a utilização da técnica de SE-AFLP como método único de tipagem para $S$. Enteritidis não deve ser recomendada. A partir disto, as técnicas de determinação da sensibilidade a antimicrobianos e fagotipificação devem ser associadas para uma melhor discriminação das amostras em estudos epidemiológicos.
Da mesma forma, outras técnicas genotípicas devem ser testadas para alcançar um índice de discriminação mais adequado.

\section{CONCLUSÃO}

A técnica de SE-AFLP mostrou um maior poder discriminatório no grupo de isolados de Salmonella Enteritidis originário de surtos de infecção alimentar em humanos e produtos de suínos do que a rep-PCR e a detecção de genes $s p v$. Dentre os iniciadores testados, o iniciador $\mathrm{C}$ gerou os melhores resultados. Entretanto a discriminação alcançada foi inferior a das técnicas fenotípicas (fagotipificação e DSA), tornando indispensável à associação de técnicas fenotípicas com a SE-AFLP para uma melhor caracterização de amostras de $S$. Enteritidis.

Agradecimentos. Agradecemos ao Conselho Nacional de Desenvolvimento Científico e Tecnológico - CNPq/Brasil, que apoiou o presente trabalho e forneceu bolsa aos autores A.F. Streck e C.S.L. Vaz.

\section{NOTAS INFORMATIVAS}

${ }^{1}$ Fermentas, EUA.

${ }^{2}$ Invitrogen, Brasil.

${ }^{3}$ Gibco, Brasil.

\section{REFERÊNCIAS}

1 Betancor L., Schelotto F., Martinez A., Pereira M., Algorta G., Rodríguez M.A., Vignoli R. \& Chabalgoity, J.A. 2004. Random amplified polymorphic DNA and phenotyping analysis of Salmonella enterica serovar Enteritidis isolates collected from humans and poultry in Uruguay from 1995 to 2002. 2004. Journal of Clinical Microbiology. 42: 1155-1162.

2 Costalunga S. \& Tondo E.C. 2002. Salmonellosis in Rio Grande do Sul, Brasil, 1997 to 1999. Brazilian Journal of Microbiology. 33: 342-346.

3 Fernandez-Cuenca F. 2004. Aplicaciones de las técnicas de PCR a la epidemiología molecular de las enfermidades infecciosas. Enfermidades Infecciosas y Microbiologia Clínica. 22:355-360.

4 de la Puente-Redondo V.A., Garcia del Blanco N., Guetiérrez-Martim C.B, Garcia-Pena F.J. \& Rodríguez Ferri E.F. 2000. Comparison of different PCR approaches for typing of Francisella tularensis strains. Journal of Clinical Microbiology. 38: 1016-1022.

5 dos Santos L.R., Nascimento V.P., de Oliveira S.D., Rodrigues D.P., dos Reis E.M.F., Seki L.M., Ribeiro A.R. \& Fernandes S.A. 2003. Phage types of Salmonella Enteritidis isolated from clinical and food samples, and from broiler carcasses in Southern Brazil. Revista do Instituto de Medicina Tropical de São Paulo. 45: 1-4.

6 Geimba M.P., Tondo E.C., de Oliveira F.A., Canal C.W. \& Brandelli A. 2004. Serological characterization and prevalence of $s p v \mathrm{R}$ genes in Salmonella isolated from foods involved in outbreaks in Brazil. Journal of Food Protection. 67: 1229-1233.

7 Gibson J.R., Slater E., Xerry J., Tompkins D.S. \& Owen R.J. 1998. Use of amplified-fragment length polymorphism technique to fingerprinting and differentiate isolates of Helicobacter pylori. Journal of Clinical Microbiology. 36: 2580-2585.

8 Hunter P.R. 1990. Reproducibility and indices of discriminatory power of microbial typing methods. Journal of Clinical Microbiology. 28: 1903-1905.

9 Janssen P., Coopman R., Huys G., Swings J., Blecker M., Vos P., Zabeau M. \& Kerster K. 1996. Evaluation of the DNA fingerprinting method AFLP as new tool in bacterial taxonomy. Microbiology. 142: 1881-1893.

10 Liebana E. 2002. Molecular tools for epidemiological investigations of S. enterica subspecies enterica infections. Research in Veterinary Science. 72: 169-175. 
11 López-Molina N., Laconcha I., Rementeria A., Audicana A., Perales I. \& Garaizar J. 1998. Typing of Salmonella enteritidis of different phage types of PCR fingerprinting. Journal of Applied Microbiology. 84: 877-882.

12 Mammina C., Giammanco G.M., Romani C., Nastasi A. 2005. Use of pulsed field gel electrophoresis (PFGE) and singleenzyme amplified fragment length polymorphism (SE-AFLP) to subtype isolates of Salmonella enterica serotype Enteritidis. Italian Journal of Public Health. 2: 23-28.

13 Mammina C., Romani C., Giammanco G.M., Luzzi I., Dionisi A.M. \& Nastasi A. 2006. Single-enzyme-amplified polymorphism (SE-AFLP) in Salmonella epidemiology. In: Proceedings of the International Symposium Salmonella and Salmonellosis (Saint-Malo, France). pp.121-123.

14 Mazurak G., Reddy H.V., Marston B.J., Haas W.J. \& Crawford J.T. 1996. DNA fingerprinting by infrequent-restriction-site amplification. Journal of Clinical Microbiology. 34: 2386-2390.

15 McLauchlin J., Ripabelli G., Brett M.M. \& Threlfall E.J. 2000. Amplified fragment length polymorphism (AFLP) analysis of Clostridium perfringens for epidemiological typing. International Journal of Food Microbiology. 56: 21-28.

16 Moreno A.M., Baccaro M.R., Ferreira A.J.P. \& de Castro A.F.P. 2003. Use of single-enzyme amplified fragment length polymorphism for typing Pasteurella multocida subsp. multocida isolates from pigs. Journal of Clinical Microbiology. 41: 1743-1746.

17 Nadvorny A., Figueiredo D.M.S. \& Schmidt V. 2004. Ocorrência de Salmonella sp. em surtos de doenças transmitidas por alimentos no Rio Grande do Sul em 2000. Acta Scientiae Veterinariae. 32: 47-51.

18 National Committee for Clinical Laboratory Standards (NCCLS). 2001. Performance standards for antimicrobial susceptibility testing. 11th informational supplement. Wayne (Approved standard M2-A7 and M7-A5). PA.

19 Nunes I.A., Helmuth R., Schroeder A., Mead G.C., Santos M.A., Solari C.A., Silva O.R. \& Ferreira A.J. 2003. Phage typing of Salmonella enteritidis from different sources in Brazil. Journal of Food Protection. 66: 324-327.

20 Oliveira S.D. 2003. Detecção de Salmonella sp. e caracterização de isolados de Salmonella Enteritidis pela presença de genes de virulência, resistência a antimicrobianos e por rep-PCR fingerprinting. 122 p. Porto Alegre. RS. Tese (Doutorado em Ciências Veterinárias) - Programa de Pós-graduação em Ciências Veterinárias, Universidade Federal do Rio Grande do Sul.

21 Oliveira S.D., Rodenbusch C.R., Ce M.C., Rocha S.L.S. \& Canal C.W. 2003. Evaluation of selective and non-selective enrichment PCR procedures for Salmonella detection. Letters in Applied Microbiology. 36: 217-221.

22 Peters T.M. \& Threfall E.J. 2001. Single-enzyme amplified fragment length polymorphism and its applicability for Salmonella epidemiology. Systematic and Applied Microbiology. 24: 400-404.

23 Rodrigue D.C., Tauxe R.V. \& Rowe B. 1990. International increase in Salmonella enteritidis: A new pandemic? Epidemiology and Infections. 105: 21-27.

24 Talon D., Cailleaux V., Thouverez M., Michel-Briand Y. 1996. Discriminatory power and usefulness of pulsed-field gel electrophoresis in epidemiological studies of Pseudomonas aeruginosa. Journal of hospital infections. 32: $135-145$.

25 Taunay A.E., Fernandez S.A., Tavechio A.T., Neves B.C., Dias A.M.G. \& Irino K. 1996. The role of public health laboratory in the problem of salmonellosis in São Paulo, Brazil. Revista do Instituto de Medicina Tropical de São Paulo. 38: 119-127.

26 Tavechio A.T., Fernandez S.A., Nevez B.C., Dias A.M.G. \& Irino K. 1996. Changing patterns of Salmonella serovars: increase of Salmonella enteritidis in São Paulo, Brazil. Revista do Instituto de Medicina Tropical de São Paulo. 38: $315-322$.

27 Thong K.L., Ngeow Y.F., Altwegg M., Navaratnam P. \& Pang T. 1995. Molecular analysis of Salmonella enteritidis by pulsed-field gel electrophoresis and ribotyping. Journal of Clinical Microbiology. 33: 1070-1074.

28 Tortora G.J., Funke B.R. \& Case C.L. 2000. Microbiologia. 6 ed. Porto Alegre: Artmed. 827 p.

29 Valsangiocomo C., Baggi F., Gaia V., Balmelli T., Peduzzi R. \& Piffaretti J. 1995. Use of amplified length polymorphism in molecular typing of Legionella pneumophila and application to epidemiological studies. Journal of Clinical Microbiology. 33: 1716-1719.

30 van der Zee A., Steer N., Thijssen E., Nelson J., van't Veen A. \& Buiting A. 2003. Use of multienzyme multiplex PCR amplified fragment length polymorphism typing in analysis of outbreaks of multiresistant Klebsiella pneumoniae in an intensive care unit. Journal of Clinical Microbiology. 41: 798-802.

31 Vaz C.S.L., Streck A.F., Tramontina T., Cardoso M.R.I., Canal C.W. 2007. Use of a modified AFLP protocol to discriminate Salmonella enterica subsp. enterica serovar Enteritidis isolates. Acta Scientiae Veterinariae. 35: 41-48.

32 Vos P., Hoger R., Bleeker M., Reijans M., van de Lee T., Hornes M., Frijters A., Pot J., Peleman J., Kuiper M. \& Zabeau M. 1995. AFLP: a new technique for DNA fingerprinting. Nucleic Acids Research. 23: 4407-4414. 